Article

\title{
Expression, Purification and Identification of CtCVNH, a Novel Anti-HIV (Human Immunodeficiency Virus) Protein from Ceratopteris thalictroides
}

\author{
Junbo Sun ${ }^{1,2}$, Yingjuan Su ${ }^{1,2, *}$ and Ting Wang ${ }^{3, *}$
}

1 State Key Laboratory of Biocontrol, School of Life Sciences, Sun Yat-sen University, Guangzhou 510275, China; E-Mail: dxxsjb@163.com

2 Institute for Technology Research and Innovation of Sun Yat-sen University, Zhuhai 519000, China

3 CAS Key Laboratory of Plant Germplasm Enhancement and Specialty Agriculture, Wuhan Botanical Garden, Chinese Academy of Sciences, Wuhan 430074, China

* Authors to whom correspondence should be addressed; E-Mails: suyj@mail.sysu.edu.cn (Y.S.); tingwang@wbgcas.cn (T.W.); Tel./Fax: +86-20-8403-6215 (Y.S.).

Received: 14 January 2013; in revised form: 3 March 2013 / Accepted: 26 March 2013 /

Published: 8 April 2013

\begin{abstract}
CVN (cyanovirin-N) is an anti-HIV protein. CVNH (cyanovirin-N homology) represents its homology. In a previous study, we first reported the full-length sequences of the $C V N H$ gene cloned from Ceratopteris thalictroides. Based on the finding, the coding sequence of $C t C V N H$ was optimized in the study, and then a pET prokaryotic expression vector was constructed. The purification and identification of CtCVNH protein were investigated, as well. SDS-PAGE analysis indicated that a $31 \mathrm{kDa}$ protein was overexpressed and mainly accumulated in the soluble fraction. Only a single protein was obtained after the Ni- nitrilotriacetic acid (NTA) affinity chromatography. The purified protein was identified to be the recombinant CtCVNH by both Western blot and peptide mass fingerprinting analysis.
\end{abstract}

Keywords: Ceratopteris thalictroides; CtCVNH; anti-HIV protein; prokaryotic expression

\section{Introduction}

AIDS is a malignant infectious disease that seriously threatens society and economics in the world. At this time, the chemotherapeutic agents, reverse transcriptase and protease, are used to treat HIV 
infection by preventing HIV replication in host cells. However, these agents cannot prevent HIV from entering the human body, and the side effects include drug resistance and damage to liver and kidney function. Therefore, it is urgent to develop novel, safe and highly effective anti-HIV agents [1,2].

Cyanovirin-N (CVN), a protein with potent anti-HIV activity, was firstly isolated from Nostoc elliposporum [3]. CVN is a 101-amino acid single-chain protein without post-translational modification. The protein contains about two 50-amino acid tandem repeats and two disulfide bond (corresponding to $\mathrm{C} 8-\mathrm{C} 22$ and $\mathrm{C} 58-\mathrm{C} 73$, respectively) [4]. The two $\mathrm{CVN}$ monomers form a domain-swapped dimmer [5]. The two carbohydrate binding sites of CVN are symmetrically located within a single protein [6]. Their affinities are very different, and one is significantly higher than the other [6]. CVN can resist detergents, denaturants organic solvents and multiple freeze-thaw cycles [3]. Even boiling at $100{ }^{\circ} \mathrm{C}$ cannot damage its anti-HIV activity [3]. CVN successfully blocks the virus-cell fusion process mediated by HIV envelope glycoprotein [3]. In vitro studies show that CVN inhibits HIV envelope-mediated cell fusion at nanomolar concentrations by interfering with the interaction between gp120 and cellular receptor CD4 [3,6-8]. Boyd et al. (1997) found that CVN possessed high activity against HIV in various target cells, including HIV-1 laboratory strains, RF, IIIB, MN, G910-6, A17, 214, SK1 and 205, G1, and HIV-1 primary isolates WEJQ, VIHU, BAKI, WOME, 89.6, Ba-L, Ada-M and SLKA. In addition, CVN has other antiviral activity, such as Ebola [9] and the influenza virus [10].

CVN homologies (cyanovirin-N homology, CVNH) are composed of the CVNH protein family, which is mainly present in bacteria, fungi and ferns. All CVNH proteins share a common fold structure with CVN $[6,11,12]$. The anti-HIV domain is extremely conserved among CVNHs, which exhibit similar antiviral activity [11]. CVNH is also indicated to be a potential natural anti-HIV protein.

In the previous study, we first reported the full-length genomic DNA of the CVNH gene from Ceratopteris thalictroides [12]. In the present, the gene encoding CVNH from $C$. thalictroides $(\mathrm{CtCVNH})$ was optimized and constructed the expression plasmid pET32a-CtCVNH. The plasmid was utilized for expression, purification and identification, which composed a solid foundation for further activity investigation of CtCVNH.

\section{Results}

\subsection{Optimization of the $\mathrm{CtCVNH}$ Gene}

The $C t C V N H$ gene was successfully optimized by deleting a signal peptide-like sequence and the stable secondary structures and regions that may block the ribosome binding site (Figure 1). The constructed plasmid, pET32a-CtCVNH, verified by DNA sequencing, was transformed into E. coli JM109. 
Figure 1. Result of sequence optimization (numbering according to the wild-type coding sequence of cyanovirin-N homology from $C$. thalictroides (CtCVNH)). UG: the wild-type coding sequence of $\mathrm{CtCVNH}$. OG: the optimized coding sequence of $\mathrm{CtCVNH}$.

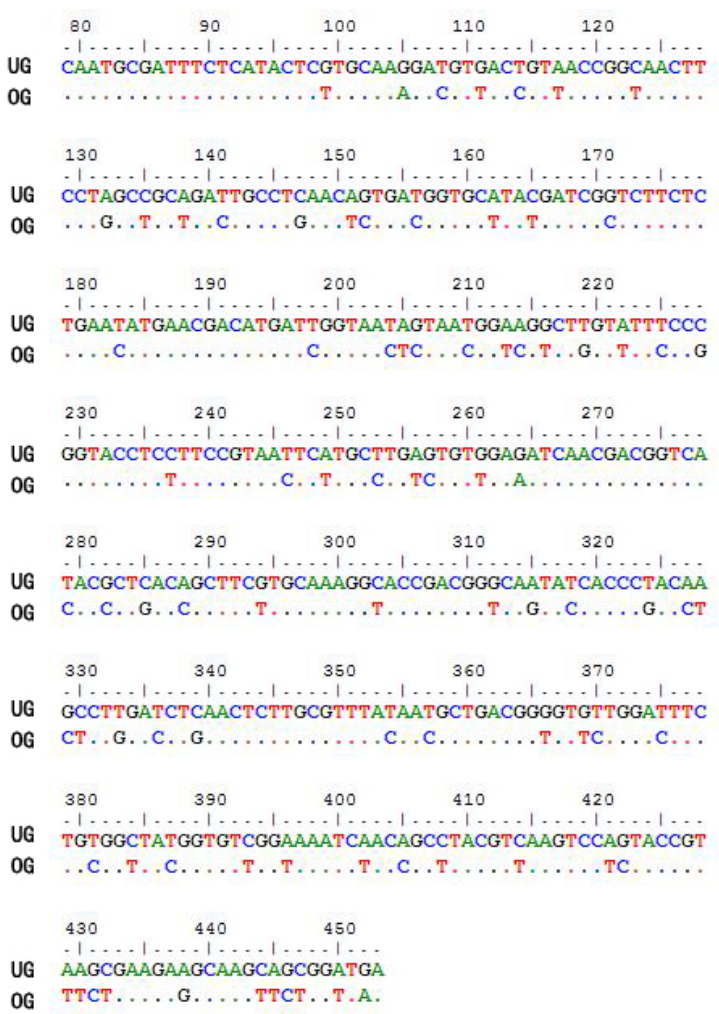

\subsection{Construction of the Expression Strain}

The optimized $C t C V N H$ gene was confirmed to be successfully inserted into the expression vector pET32 (+). The recombinant plasmid pET32a-CtCVNH digested with EcoR I and Hind III corresponding to $5900 \mathrm{bp}$ and $375 \mathrm{bp}$ bands, respectively (Figure 2). The inserted DNA sequence was identical to the optimized $C t C V N H$ gene after having been successfully transformed into Rosetta 2 (DE3).

Figure 2. Restriction enzyme digestion of the recombinant plasmid pET32a-CtCVNH by EcoR I and Hind III. $\mathrm{M}_{1}$ : $\lambda$-Hind III digest DNA marker. 1, pET32a (+); 2, pET32a-CtCVNH; $\mathrm{M}_{2}, 100$ bp DNA ladder marker.

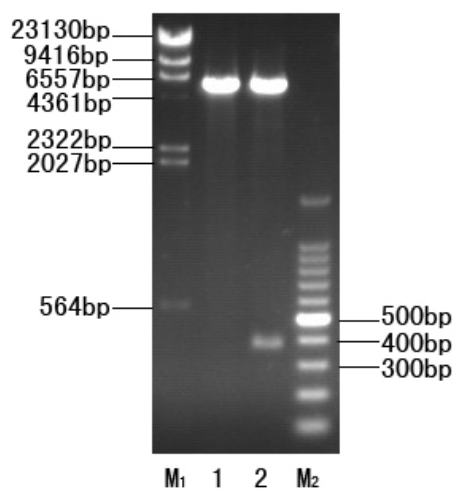




\subsection{Protein Expression and Solubility Analysis}

Recombinant $C t C V N H$ gene was expressed in Rosetta 2 (DE3) induced by $0.5 \mathrm{mM}$ IPTG for $2 \mathrm{~h}$. The recombinant protein corresponded to $31 \mathrm{kDa}$, suggesting an efficient expression of the optimized CtCVNH gene. Solubility analysis indicated that CtCVNH protein accumulated mainly in the soluble fraction (Figure 3A).

Figure 3. Coomassie-stained SDS-PAGE gel (A) and Western blot (B) showing the expression profile of CtCVNH. M, low molecular protein marker; Lane 1, sample before induction; Lane 2, sample after induced; Lane 3, supernatant; Lane 4, inclusion body; Lane 5, purified sample.
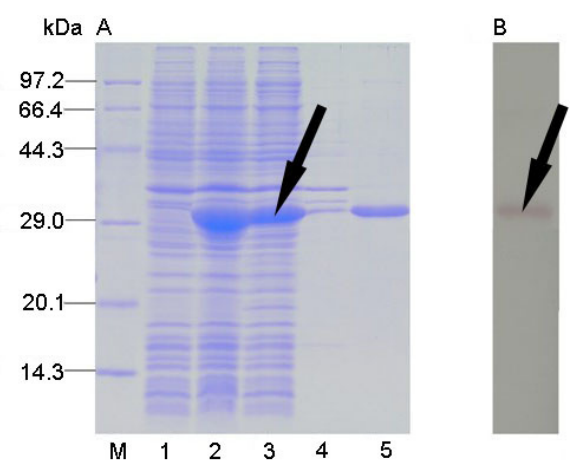

\subsection{Protein Purification and Western Blot Analysis}

A single protein band with correct molecular weights from the supernatant was obtained by Ni-NTA resin purification (Figure 3A). The results also indicated that the recombinant CtCVNH is of high purity. The purified proteins were further confirmed by Western blot with mouse anti-His tag monoclonal antibody and goat anti-mouse IgG antibody conjugated to alkaline phosphatase, which detected a single band with correct molecular weights (Figure 3B).

\subsection{PMF Analysis and Molecular Weight Determination}

The recombinant protein matched well with the $C$. richardii CVNH data set (GenBank Accession No. BQ087187, Score 282, $p<0.05)$. The $m / z$ ratios yielded experimental molecular weights of 31,049.74 Da for recombinant protein, which was close to the predicted mass of 31,048.83 Da (Figure 4).

Figure 4. The molecular weight of recombinant CtCVNH determined by MATOL-TOF/MS.

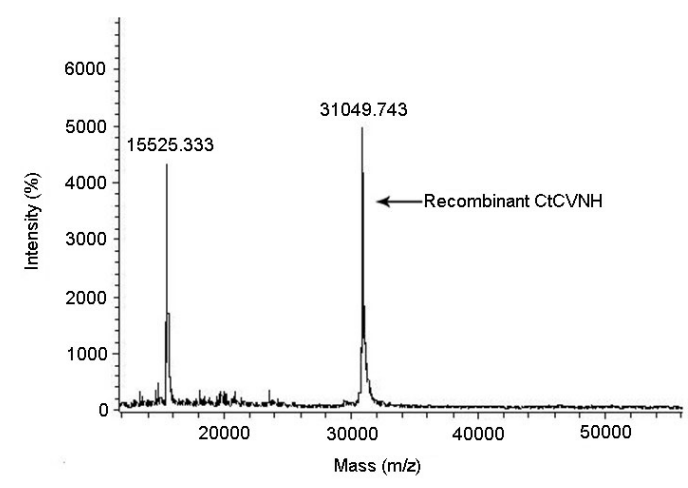




\section{Discussion}

Escherichia coli is one of the most favorite hosts for protein heteroexpression. It is widely used in protein expression, due to its low cost, fast growth and high yield [13]. Although many proteins have been successfully expressed in E. coli, most of them were expressed as inactive inclusion bodies [13]. The purification of inclusion bodies is considered labor-intensive, time-consuming and not cost-effective [13]. The pET systems are very powerful for high efficient prokaryotic expression under the control of strong bacteriophage $\mathrm{T} 7$ transcription and translation signals. Vector pET32a $(+)$ is one of the pET systems that permits target genes to be fused to trxA for high levels of expression with good solubility [14].

In a previous study, we constructed a recombinant pET32a $(+)$ plasmid to produce wild-type $\mathrm{CtCVNH}$. The recombinant protein CtCVNH could be expressed in E. coli. Several E. coli rare codons were found in the coding sequence of $\mathrm{CtCVNH}$, two of which were in tandem. In order to avoid translation errors caused by rare codons, E. coli strain Rosetta 2 (DE3), which could supply tRNAs recognizing rare codons, was used as the expression host for recombinant CtCVNH. However, two expression proteins were detected, corresponding to $34 \mathrm{kDa}$ and $23 \mathrm{kDa}$, respectively, which could not be separated by affinity chromatography. The Peptide Mass Fingerprinting (PMF) and bioinformatics analysis indicated that the $23 \mathrm{kDa}$ protein was identical to the $\mathrm{N}$-terminus part of the target protein. We inferred that the $23 \mathrm{kDa}$ protein was a partial product resulting from premature termination $[15,16]$.

In order to obtain the single recombinant CtCVNH protein, the coding sequence of the CtCVNH protein was optimized. Codons that might affect protein expression were replaced with E. coli preferred codons. The protein of the optimized gene was expressed as described above. An obvious expression band about $31 \mathrm{kDa}$ could be detected. More importantly, only a target protein was detected, which was purified through affinity chromatography. The purified protein was further confirmed by Western blot and PMF to be the recombinant CtCVNH. Due to Trx tag attached to the $N$-terminus of the protein, the target protein can be directly purified from the supernatant. Therefore, the denaturation and refolding of the protein can be avoided. Furthermore, as a native protein of E. coli, Trx can protect CtCVNH from protease degradation [17].

\section{Experimental Section}

\subsection{Experimental Materials}

Plasmid pET32a $(+)$ and E. coli strain Rosetta 2 (DE3) were used as hosts for the expression of CVNH. $\lambda$-Hind III digest DNA marker, 100 bp DNA ladder marker, protein molecular weight marker (low) and restriction enzymes EcoR I and Hind III were supplied by TaKaRa (Dalian, China). An AxyPrepTM Plasmid Miniprep Kit was purchased from Axygen (Hangzhou, China). Ni-NTA His·Bind Resin was obtained from Novagen (San Diego, CA, USA). Amicon Ultra-15 centrifugal filter units $(10 \mathrm{kDa})$ were acquired from Millipore (Billerica, MA, USA). Mouse anti-His6 monoclonal antibody and goat anti-mouse IgG antibody conjugated to alkaline phosphatase (AP) were purchased from CWBIC (Beijing, China). All other chemicals were of analytical grade and obtained from commercial sources. 


\subsection{Sequence Optimization and Construct Design}

Based on the previous study, the gene encoding CtCVNH was obtained from its full length sequences [12]. First, the signal peptide was predicted using SignalP 4.0 [18] (http://www.cbs.dtu.dk/services/SignalP/). Second, after the signal peptide was deleted, the CtCVNH gene was optimized by Jcat, which is a web-based program [19] (http://www.jcat.de/). Third, the optimized $C t C V N H$ gene was inserted into plasmid pET32a ( + ) between the digestive sites, EcoR I and Hind III. Fourth, the secondary structure of the mRNA of the expression plasmid was predicted by RNAfold (http://rna.tbi.univie.ac.at/) [20]. Fifth, the CtCVNH gene was further optimized to eliminate the stable secondary structures and regions that might block the ribosome binding site. Finally, the finial optimized CtCVNH gene introduced to the sites of EcoR I and Hind III was synthesized in TaKaRa, which was ligated to the similarly digested expression vector, pET32a $(+)$. The constructed recombinant plasmid pET32a-CtCVNH was transformed into E. coli JM109.

\subsection{Construction of the Expression Strain}

Plasmid DNA from the successful clones of pET32a-CtCVNH was extracted using the AxyPrep ${ }^{\mathrm{TM}}$ Plasmid Miniprep Kit and verified by restriction enzyme digestion. The plasmid DNA was transformed into Rosetta 2 (DE3) E. coli cells for expression of the recombinant protein. The sequence of the cloned gene was verified by sequencing. The plasmid was stored at $-80^{\circ} \mathrm{C}$ for glycerol stocks.

\subsection{Expression of Recombinant Protein and Solubility Analysis}

A single positive pET32a-CtCVNH clone was inoculated into $10 \mathrm{~mL}$ LB culture containing ampicillin $(100 \mu \mathrm{g} / \mathrm{mL})$ and chloramphenicol $(34 \mu \mathrm{g} / \mathrm{mL})$ and incubated overnight at $37{ }^{\circ} \mathrm{C}$. The overnight culture was inoculated into $300 \mathrm{~mL}$ fresh $\mathrm{LB}$ culture containing ampicillin and chloramphenicol at a ratio of $1: 100$ and grown at $37{ }^{\circ} \mathrm{C}$ with shaking $(220 \mathrm{rpm})$. When the $\mathrm{OD}_{600}$ reached approximately $0.6-1.0$, isopropyl $\beta$-D-1-thiogalactopyranoside (IPTG) was added with a final concentration of $0.2 \mathrm{mM}$. The culture was incubated at $37^{\circ} \mathrm{C}$ for an additional $2 \mathrm{~h}$, and the protein was autoinduced to be expressed during this period.

\subsection{Protein Purification}

The cells were harvested from the $300 \mathrm{~mL}$ culture by centrifugation at $8,000 \times \mathrm{g}$ for 6 min at $4{ }^{\circ} \mathrm{C}$. Then, the cells were resuspended in $15 \mathrm{~mL}$ of lysis buffer $(20 \mathrm{mM}$ Tris-HCL, pH 7.5), and then lysed on ice by sonication at $30 \mathrm{~W}$ for 50 cycles ( $10 \mathrm{~s}$ working, $15 \mathrm{~s}$ free). The pellet and the supernatant were separated by centrifugation at $14,000 \times g$ for $20 \mathrm{~min}$. The pellet was resuspended in $30 \mathrm{~mL}$ buffer with $20 \mathrm{mM}$ Tris-HCl (pH 7.5) to dissolve the expression protein. Each fraction was analyzed using $15 \%$ standard SDS-PAGE gel electrophoresis. The recombinant proteins were purified using Novagen Ni-NTA chromatography (San Diego, CA, USA), according to the manufacturer's protocols. Firstly, Ni-NTA His $\cdot$ Bind Resin was washed with 5-times the volume of $20 \mathrm{mM}$ Tris (pH 7.5) and balanced overnight at $4{ }^{\circ} \mathrm{C}$. The sample was loaded onto the column after passing through a $0.45 \mu \mathrm{m}$ syringe filter. Secondly, unbound proteins were removed with 10-times the volume of Buffer I (10 mM Tris- $\mathrm{HCl}, 250 \mathrm{mM} \mathrm{NaCl}, 2.5 \mathrm{mM}$ imidazole, $\mathrm{pH}$ 7.5), whereas the recombinant proteins were eluted 
with 5-times the volume of Buffer I (containing 50, 100 and $500 \mathrm{mM}$ imidazole). Finally, the column was eluted with 5-times the volume of Buffer II $(20 \mathrm{mM}$ Tris- $\mathrm{HCl}, 500 \mathrm{mM} \mathrm{NaCl}, 100 \mathrm{mM}$ EDTA, $\mathrm{pH} 7.5)$.

\subsection{Western Blot Analysis}

Electrophoretic separation of protein preparations was carried out using SDS-PAGE. The proteins were transferred to nitrocellulose membranes by electroblotting. The membrane was blocked with $3 \%$ BSA at room temperature for $2 \mathrm{~h}$ and was incubated overnight with mouse anti-His tag monoclonal antibody (1:5,000). After washing, the membrane was incubated with goat anti-mouse IgG antibody conjugated to alkaline phosphatase $(1: 10,000)$ for $2 \mathrm{~h}$ at room temperature.

\subsection{Peptide Mass Fingerprinting Analysis and Molecular Weight Determination}

The Peptide Mass Fingerprinting (PMF) and Matrix Assisted Laser Desorption/Ionization Time of Flight Mass Spectrometry (MALDI-TOF/MS) were performed on MLtraflex MALDI-TOF/TOF (Bruker Corporation, Billerica, MA, USA) in the Experimental Center, School of Life Science, Sun Yat-sen University (Guangzhou, China). For PMF, the purified recombinant protein was electrophoresed on SDS-PAGE, followed by Coomassie staining. The protein band was carefully excised, destained and digested using trypsin. Data were analyzed using software Mascot v2.3 by searching for $\mathrm{CVNH}$ in the NCBI non-redundant database. For MALDI-TOF/MS, $5 \mathrm{~mL}$ of purified protein was added to the Amicon Ultra-15 centrifugal filter units $(10 \mathrm{kDa})$ and spun at $3,500 \times \mathrm{g}$ for $40 \mathrm{~min}$ at $4{ }^{\circ} \mathrm{C}$. Ten milliliters of ultra-pure water were added and spun at 3,500× $\mathrm{g}$ for $60 \mathrm{~min}$ at $4{ }^{\circ} \mathrm{C}$ and repeated once. The samples were then analyzed under high vacuum with a $25 \mathrm{kV}$ beam. All MS spectra were acquired in positive-ion mode with 200 laser pulses per sample spot.

\section{Conclusions}

This study is the first to report the construct of a pET prokaryotic expression vector based on the optimized coding sequence of $C t C V N H$. CVNH protein can be successfully expressed, purified and identified, which lays a solid foundation for further activity investigation of CtCVNH. In another experiment, we optimized the culture condition of $\mathrm{CVNH}$ protein expression, including the type and the ingredients of the culture medium, initial $\mathrm{pH}$, OD value, induction agents and concentrations, induction time and expression time, respectively [21]. In the future, we will explore the biologically active, clinical trials, large-scale preparation and commercialization of CtCVNH.

\section{Acknowledgments}

The authors thank the Yongjin Liu of School of Life Sciences, Sun Yat-sen University, for assistance with PMF analysis and molecular weight determination. This work was supported by the National Natural Science Foundation of China (30771763, 30970290 and 31070594), the National Natural Science Foundation of Guangdong Province (S2012010010502), the Knowledge Innovation Program of the Chinese Academy of Sciences (KSCX2-EW-J-20, KSCX2-YW-Z-0940), the Opening Fund of Laboratory, Sun Yat-sen University (KF201128), the Key Pilot Project of Department of 
Science and Technology of Guangdong Province, China (2011B031700007, 2012B010300009), the Key Pilot Project of the Department of Science and Technology of Guangzhou, China (2010J-E101), the Guangdong Key Laboratory of Plant Resources (plant01k13) and the Scientific Research Fund, Hongda Zhang, Sun Yat-sen University.

\section{Conflict of Interest}

The authors declare no conflict of interest.

\section{References}

1. Clercq, E.D. Anti-HIV drugs: 25 compounds approved within 25 years after the discovery of HIV. Int. J. Antimicrob. Agents 2009, 33, 307-320.

2. Arts, E.J.; Hazuda, D.J. HIV-1 antiretroviral drug therapy. Cold Spring Harb. Perspect. Med. 2012, 2, a007161.

3. Boyd, M.R.; Gustafson, K.R.; Mcmahon, J.B.; Shoemaker, R.H.; O’Keefe, B.R.; Mori, T.; Gulakowski, R.J.; Wu, L.; Rivera, M.I.; Laurencot, C.M.; et al. Discovery of cyanovirin-N, a novel human immunodeficiency virus-inactivating protein that binds viral surface envelope glycoprotein gp120: Potential applications to microbicide development. Antimicrob. Agents Chemother. 1997, 41, 1521-1530.

4. Gustafson, K.R.; Sowder, R.C.; Henderson, L.E.; Cardellina, J.H.; McMahon, J.B.; Rajamani, U.; Pannell, L.K.; Boyd, M.R. Isolation, primary sequence determination, and disulfide bond structure of cyanovirin-N, an anti-HIV (human immunodeficiency virus) protein from the cyanobacterium Nostoc ellipsosporum. Biochem. Biophs. Res. Commun. 1997, 238, 223-228.

5. Yang, F.; Bewley, C.A.; Louis, J.M.; Gustafson, K.R.; Boyd, M.R.; Gronenborn, A.M.; Clore, G.M.; Wlodawer, A. Crystal structure of cyanovirin-N, a potent HIV-inactivating protein, shows unexpected domain swapping. J. Mol. Biol. 1999, 288, 403-412.

6. Bewley, C.A.; Otero-Quintero, S. The potent anti-HIV protein cyanovirin-N contains two novel carbohydrate binding sites that selectively bind to $\mathrm{Man}_{8}$ D1D3 and Man 9 with nanomolar affinity: Implications for binding to the HIV envelope protein gp120. J. Am. Chem. Soc. 2001, 123, 3892-3902.

7. Bewley, C.A.; Gustafson, K.R.; Boyd, M.R.; Covell, D.G.; Bax, A.; Clore, G.M.; Gronenborn, A.M. Solution structure of cyanovirin-N, a potent HIV-inactivating protein. Nat. Struct. Biol. 1998, 5, 571-578.

8. Bolmstedet, A.J.; O’Keefe, B.R.; Shenoy, S.R.; McMahon, J.B.; Boyd, M.R. Cyanovirin-N defines a new class of antiviral agent targeting $N$-linked, high-mannose glycans in an oligosaccharide-specific manner. Mol. Pharmacol. 2005, 59, 949-954.

9. Barrientos, L.G.; O’Keefe, B.R.; Bray, M.; Sanchez, A.; Gronenborn, A.M.; Boyd, M.R. Cyanovirin-N binds to the viral surface glycoprotein, $\mathrm{GP}_{1,2}$ and inhibits infectivity of Ebola virus. Antiviral. Res. 2003, 58, 47-56.

10. O’Keefe, B.R.; Smee, D.F.; Turpin, J.A.; Saucedo, C.J.; Gustafson, K.R.; Mori, T.; Blakeslee, D.; Buckheit, R.; Boyd, M.R. Potent anti-influenza activity of cyanovirin-N and interactions with viral hemagglutinin. Antimicrob. Agents Chemother. 2003, 47, 2518-2525. 
11. Percudani, R.; Montanini, B.; Ottonello, S. The anti-HIV cyanovirin-N domain is evolutionarily conserved and occurs as a protein module in eukaryotes. Proteins 2005, 60, 670-6478.

12. Qi, X.Q.; Yang, Y.X.; Su, Y.J.; Wang, T. Molecular cloning and sequence analysis of cyanovirin-N homology gene in Ceratopteris thalictroides. Am. Fern J. 2009, 99, 78-92.

13. Baneyx, F. Recombinant protein expression in Escherichia coli. Curr. Opin. Biotechnol. 1999, 10, 411-421.

14. Wong, S.H. Cloning of flavin reductase into pET32a $(+)$ expression vector lacking the thioredoxin A tag to study solubility of EDTA monooxygenase A in overexpression systems. J. Exp. Microbiol. Immunol. 2005, 8, 59-66.

15. Burgess-Brown, N.A.; Sharma, S.; Sobot, F.; Loenarz, C.; Oppermann, U.; Gileadi, O. Codon optimization can improve expression of human genes in Escherichia coli: A multi-gene study. Protein Expr. Purif. 2008, 59, 94-102.

16. Han, J.H.; Choi, Y.S.; Kim, W.J.; Jeon, Y.H.; Lee, S.K.; Lee, B.J.; Ryu, K.S. Codon optimization enhances protein expression of human peptide deformylase in E. coli. Protein Expr. Purif. 2010, 70, 224-230.

17. Stewart, E.J.; Aslund, F.; Beckwith, J. Disulfide bond formation in the Escherichia coli cytoplasm: An in vivo role reversal for the thioredoxins. EMBO J. 1998, 17, 5543-5550.

18. Petersen, T.N.; Brunak, S.; von Heijne, G.; Nielsen, H. SignalP 4.0: Discriminating signal peptides from transmembrane regions. Nat. Methods 2011, 8, 785-786.

19. Grote, A.; Hiller, K.; Scheer, M.; Munch, R.; Nortemann, B.; Hempet, D.C.; Jahn, D. JCat: A novel tool to adapt codon usage of a target gene to its potential expression host. Nucleic Acids Res. 2005, 33, 526-531.

20. Gruber, A.R.; Lorenz, R.; Bernhart, S.H.; Neubock, R.; Hofacker, I.L. The Vienna RNA websuite. Nucleic Acids Res. 2008, 36, 70-74.

21. Li, N.; Sun, J.B.; W, J.; Q, L.G.; W, T.; Su, Y.J. Optimization for culture condition of CVNH in Escherichia coli, A novel anti-HIV (Human Immunodeficiency Virus) protein (in Chinese). Acta Sci. Nat. Univ. Sunyatseni 2013, 52, 90-96.

(C) 2013 by the authors; licensee MDPI, Basel, Switzerland. This article is an open access article distributed under the terms and conditions of the Creative Commons Attribution license (http://creativecommons.org/licenses/by/3.0/). 